\title{
ORT.26 - Host-genetic combination based on IFNL3/IFNL4 polymorphism with other prognostic variables increases sustained response in antiviral therapy with pegylated interferon alpha
}

Andréa M. V. da Silva ${ }^{1 \star}$; Lucia Elena Alvarado-Arnez²; Tamiris Azamor ${ }^{1}$; Leonardo Ribeiro Batista-Silva ${ }^{1}$; Thyago Leal Calvo ${ }^{3}$; Jane da Silva ${ }^{1}$; Ana Carolina Magalhães Andrade ${ }^{1}$; Janaina Reis Xavier ${ }^{1}$; Denise C. S. Matos ${ }^{1}$; Milton Ozorio Moraes ${ }^{3}$.

1Fiocruz/Bio-Manguinhos;

2Coordinación Nacional de Investigación, Universidad Privada Franz Tamayo - Bolívia; 3Fiocruz/IOC.

Introduction: Chronic hepatitis $\mathrm{C}(\mathrm{CHC})$ is the main cause of liver disease and hepatocellular carcinoma progress worldwide. Although direct-acting antivirals (DAAs) are the treatment of choice, there are still special cases in which peginterferon alfa (Peg-IFN $\alpha$ ) therapy should be used, such as children and patients infected with hepatitis Virus C (HCV) genotype 3 (GT3). Over the past few years, many studies have identified predictive factors for sustained virologic response (SVR), while host or viral factors are related to a successful Peg-IFN $\alpha$ and ribavirin (RBV) therapy. Single nucleotide polymorphisms (SNPs) in the interferon lambda 3 e 4 region (IFNL3/4) are wellestablished as prognostic markers after Pegylated-Interferon-alpha/ribavirin (Peg-IFN- $\alpha /$ RBV) treatment for CHC. The SNPs rs12979860, rs8109886 and rs8099917 are representative of the IFNL3/4 locus, associated with SVR, in the Brazilian population. So, the combination of predictive factors to obtain SVR could aid personalized and appropriate treatment for the population. This work was submitted to Research Ethics Committee of CAAE: 46065015.6.0000.5248.

Objective: Evaluate the contribution of host genetics, and other prognostic variables in $\mathrm{CHC}$ patients with treated with Peg-IFN $\alpha$, in SVR.

Methodology: Three IFNL3-IFNL4 SNPs (rs12979860, rs8109886 and rs8099917) were genotyped by allelic discrimination in 632 chronic hepatitis C patients infected GT1, GT2 or GT3 treated with Peg-IFN- $\alpha /$ RBV, samples from a phase II/III randomized double-blind clinical trial. Serum samples of a subgroup of patients and healthy volunteers were used to measure CCL3, CCL4 and CXCL10, using liquid bead microarray assay.

Results: Individually, either rs12979860-CC, rs8109886-CC or rs8099917-TT genotypes are predictive markers of SVR. The combination of these three genotypes (CC-CC-TT) increased to $73 \%$ at GT1 and $83 \%$ at GT3 of the rate of SVR. In contrast, patients infected with GT1 and homozygous for risk genotypes (TT-AA-GG) only $18 \%$ achieved SVR. Focusing on personalised therapy, patients with CC-CC-TT haplotype, HCV GT1-infected with viral load $<5,9 \mathrm{UI} / \mathrm{mL}$ Log10 showed an increased $82 \%$ SVR, while HCV GT2/3-infected patients with viral load $<5,9 \mathrm{UI} / \mathrm{mL} \log 10$ or F0 and F1 showed at least $93 \%$ and $96 \%$ SVR, respectively. The levels of the chemokines CCL3, CCL4 decreased after starting treatment, whereas, an increase of CXCL10 levels was observed in the first week, with a decreased over the course of the treatment. Higher levels of these chemokines presented an association with non-responsiveness after treatment.

Conclusion: The analysis of rs12979860, rs8109886 and rs8099917 genotypic combination might be an approach when Peg-IFN- $\alpha /$ RBV therapy is necessary since cost-benefit for individuals carrying CC-CC-TT genotypic combination is very high, especially in countries in which this is the standard treatment, contributing to public health efforts to eradicate disease.

Keywords: Chronic C Hepatitis; Polymorphism; Lambda Interferon 